PROCEEDINGS OF THE

AMERICAN MATHEMATICAL SOCIETY

Volume 130 , Number 10, Pages 3025-3031

S 0002-9939(02)06428-6

Article electronically published on March 15, 2002

\title{
TRIPLES OF ARRANGEMENTS AND LOCAL SYSTEMS
}

\author{
DANIEL C. COHEN
}

(Communicated by Ronald A. Fintushel)

\begin{abstract}
For a triple of complex hyperplane arrangements, there is a wellknown long exact sequence relating the cohomology of the complements. We observe that this result extends to certain local coefficient systems, and use this extension to study the characteristic varieties of arrangements. We show that the first characteristic variety may contain components that are translated by characters of any order, thereby answering a question of A. Suciu.
\end{abstract}

\section{INTRODUCTION}

Let $\mathcal{A}=\left\{H_{1}, \ldots, H_{n}\right\}$ be a hyperplane arrangement in $\mathbb{C}^{\ell}$, with complement $M(\mathcal{A})=\mathbb{C}^{\ell} \backslash \bigcup_{j=1}^{n} H_{j}$. The cohomology of $M(\mathcal{A})$ with coefficients in a complex rank one local system has been the subject of considerable recent interest, with applications in the theory of multivariable hypergeometric functions [1, 7, 15] and mathematical physics [18]. The study of such local systems is also intimately related to a number of algebraic, combinatorial, and topological invariants of arrangements.

Note that $M(\mathcal{A})$ has the homotopy type of an $\ell$-dimensional cell complex. The set of rank one local systems on $M(\mathcal{A})$ may be realized as the complex torus $\left(\mathbb{C}^{*}\right)^{n} \cong$ $H^{1}\left(M(\mathcal{A}) ; \mathbb{C}^{*}\right)$, with coordinates $\left(z_{1}, \ldots, z_{n}\right), z_{j}$ corresponding to $H_{j} \in \mathcal{A}$. A point $\mathbf{t} \in\left(\mathbb{C}^{*}\right)^{n}$ determines a representation $\rho: \pi_{1}(M(\mathcal{A})) \rightarrow \mathbb{C}^{*}$ given by $\gamma_{j} \mapsto t_{j}$ for any meridian loop $\gamma_{j}$ about the hyperplane $H_{j}$ of $\mathcal{A}$, and a corresponding rank one local system $\mathcal{L}_{\mathbf{t}}$ on $M(\mathcal{A})$. For sufficiently generic $\mathbf{t}$, the cohomology $H^{q}\left(M(\mathcal{A}) ; \mathcal{L}_{\mathbf{t}}\right)$ vanishes, except possibly in dimension $\ell$. Those $\mathbf{t}$ for which the cohomology does not vanish comprise the characteristic varieties

$$
\Sigma_{m}^{q}(\mathcal{A})=\left\{t \in\left(\mathbb{C}^{*}\right)^{n} \mid \operatorname{dim} H^{q}\left(M(\mathcal{A}) ; \mathcal{L}_{\mathbf{t}}\right) \geq m\right\} .
$$

These varieties are homotopy-type invariants of $M(\mathcal{A})$, which inform on the homology of certain abelian covers [9, 16], and also on the structure of the second nilpotent quotient of the fundamental group [13]. It follows from work of Arapura [2] that the characteristic varieties, or cohomology support loci, are unions of torsion-translated subtori of $\left(\mathbb{C}^{*}\right)^{n}$ in more general circumstances.

There is a combinatorial cohomology theory, with corresponding support loci, that is closely related to the study of (rank one) local systems on complements

Received by the editors April 9, 2001 and, in revised form, May 23, 2001.

2000 Mathematics Subject Classification. Primary 32S22; Secondary 52C35, 55N25, 14M12.

Key words and phrases. Arrangement, local system, characteristic variety, translated torus.

Partially supported by Louisiana Board of Regents grant LEQSF(1999-2002)-RD-A-01 and by National Security Agency grant MDA904-00-1-0038. 
of arrangements. We use notation and results from [14. Let $A(\mathcal{A})$ be the OrlikSolomon algebra of $\mathcal{A}$ generated by the 1 -dimensional classes $a_{j}, 1 \leq j \leq n$. It is the quotient of the exterior algebra generated by these classes by a homogeneous ideal, so is a finite dimensional graded $\mathbb{C}$-algebra. There is an isomorphism of graded algebras $A(\mathcal{A}) \cong H^{*}(M ; \mathbb{C})$. In particular, $A^{1}(\mathcal{A}) \cong H^{1}(M ; \mathbb{C}) \cong \mathbb{C}^{n}$, with coordinates $\left(y_{1}, \ldots, y_{n}\right), y_{j}$ corresponding to $H_{j} \in \mathcal{A}$. A point $\boldsymbol{\lambda} \in \mathbb{C}^{n}$ determines an element $a_{\boldsymbol{\lambda}}=\sum_{j=1}^{n} \lambda_{j} a_{j}$ in $A^{1}(\mathcal{A})$, and multiplication by $a_{\boldsymbol{\lambda}}$ provides $A(\mathcal{A})$ with the structure of a cochain complex. For sufficiently generic $\boldsymbol{\lambda}$, the cohomology $H^{q}\left(A(\mathcal{A}), a_{\boldsymbol{\lambda}} \wedge\right)$ vanishes, except possibly in dimension $\ell$. Those $\boldsymbol{\lambda}$ for which the cohomology does not vanish comprise the resonance varieties

$$
\mathcal{R}_{m}^{q}(\mathcal{A})=\left\{\boldsymbol{\lambda} \in \mathbb{C}^{n} \mid \operatorname{dim} H^{q}\left(A(\mathcal{A}), a_{\boldsymbol{\lambda}} \wedge\right) \geq m\right\} .
$$

These varieties are isomorphism-type invariants of $A(\mathcal{A})$, which, if $\mathcal{A}$ is central, inform on the structure (through rank 3) of the matroid of $\mathcal{A}$ [5]. It follows from Theorem 1 below that the resonance varieties are unions of linear subspaces of $\mathbb{C}^{n}$.

Recent work of a number of authors [3, 4, 10, 11, 12] establishes the following relationship between the characteristic and resonance varieties of an arrangement.

Theorem 1. Let $\mathcal{A}$ be an arrangement of n hyperplanes in $\mathbb{C}^{\ell}$. Then for each $q$ and $m$, the resonance variety $\mathcal{R}_{m}^{q}(\mathcal{A})$ coincides with the tangent cone of the characteristic variety $\Sigma_{m}^{q}(\mathcal{A})$ at the point $\mathbf{1}=(1, \ldots, 1) \in\left(\mathbb{C}^{*}\right)^{n}$.

This result shows that the structure of the characteristic varieties is largely combinatorial. Components of $\Sigma_{m}^{q}(\mathcal{A})$ containing $\mathbf{1}$ are determined by the resonance varieties of the Orlik-Solomon algebra $A(\mathcal{A})$. In turn, $A(\mathcal{A})$ is determined by the combinatorics of $\mathcal{A}$, namely the intersection poset $L(\mathcal{A})$; see [14. The best understood characteristic and resonance varieties are those for the first cohomology, $\Sigma_{m}(\mathcal{A}):=\Sigma_{m}^{1}(\mathcal{A})$ and $\mathcal{R}_{m}(\mathcal{A}):=\mathcal{R}_{m}^{1}(\mathcal{A})$. We refer to [4, 5, 10 12] and the surveys 6, 19. In particular, explicit combinatorial descriptions of the resonance varieties $\mathcal{R}_{m}(\mathcal{A})$, and hence of the components of $\Sigma_{m}(\mathcal{A})$ containing $\mathbf{1}$, are known.

However, as noted by Yuzvinsky [19. §8.4], "relations between the resonance and respective characteristic varieties are still mysterious," Theorem 1 notwithstanding. There are arrangements for which the characteristic varieties contain components which do not pass through $\mathbf{1}$, so are not a priori detectable by combinatorial means. The first such example was found in 4. The most striking examples of this phenomenon were found recently by Suciu [17, who exhibits arrangements for which the first characteristic variety contains positive-dimensional translated components.

This discovery prompted a number of questions in [17]. One can ask, for instance, are these translated components combinatorially determined? what are the possible dimensions? what are the possible orders of translation? In this note, we answer the last of these questions as follows.

Theorem 2. For any positive integer $r \geq 2$, there is a hyperplane arrangement $\mathcal{A}$ whose first characteristic variety $\Sigma_{1}(\mathcal{A})$ contains positive-dimensional components which are translated by characters of order $r$.

This result is established in Section 3 Call a component of $\Sigma_{m}(\mathcal{A})$ essential if it is not contained in a subtorus $z_{j}=1$, in the coordinates for $\left(\mathbb{C}^{*}\right)^{n}$ specified above. Analogously, call a component of $\mathcal{R}_{m}(\mathcal{A})$ essential if it is not contained in a hyperplane $y_{j}=0$, in the chosen coordinates for $\mathbb{C}^{n}$. We exhibit a family of arrangements $\left\{\mathcal{D}_{r}\right\}_{r \geq 2}$, whose first characteristic varieties have essential components that are translated as asserted. The first member of this family, $\mathcal{D}_{2}$, is the 
"deleted $B_{3}$ arrangement," the principal example found by Suciu 17]. Viewing the $\mathrm{B}_{3}$ Coxeter arrangement as the reflection arrangement associated to the full monomial group $G(2,1,3)$, one is led to study the characteristic varieties of deletions $\mathcal{D}_{r}$ of the arrangements associated to the full monomial groups $G(r, 1,3)$ for any $r$.

For small $r$, we found translated components in the characteristic varieties $\Sigma_{1}\left(\mathcal{D}_{r}\right)$ by direct calculation. This note stems from an attempt to find a conceptual, and perhaps combinatorial, explanation for the existence of these translated components. To this end, we revisit a standard construction in arrangement theory, that of a deletion-restriction triple, in the context of local systems. In Section 2, we observe that a well-known exact sequence relating the (constant coefficient) cohomology of the complements of the arrangements in a triple extends to certain local systems. This observation facilitates the proof of Theorem 2 .

\section{TRIPLES}

Let $\mathcal{A}$ be a hyperplane arrangement in $\mathbb{C}^{\ell}$. The choice of a distinguished hyperplane $H$ in $\mathcal{A}$ gives rise to a triple of arrangements $\left(\mathcal{A}, \mathcal{A}^{\prime}, \mathcal{A}^{\prime \prime}\right)$, where $\mathcal{A}^{\prime}=\mathcal{A} \backslash\{H\}$ and $\mathcal{A}^{\prime \prime}$ is the arrangement in $H \cong \mathbb{C}^{\ell-1}$ with hyperplanes $\left\{K \cap H \mid K \in \mathcal{A}^{\prime}\right\}$.

Let $M=M(\mathcal{A}), M^{\prime}=M\left(\mathcal{A}^{\prime}\right)$, and $M^{\prime \prime}=M\left(\mathcal{A}^{\prime \prime}\right)$ be the complements of the arrangements in a triple. These complements are related by

$$
M=M^{\prime} \backslash\left(M^{\prime} \cap H\right) \text { and } M^{\prime \prime}=M^{\prime} \cap H .
$$

The interrelations among the cohomology (rings) of $M, M^{\prime}$, and $M^{\prime \prime}$ have been the subject of much study; see [8, 14, 19]. For instance, the following is well-known.

Proposition 3 ([14, Cor. 5.81]). For a triple of complex hyperplane arrangements, there is a long exact sequence in cohomology

$$
\cdots \rightarrow H^{q}\left(M^{\prime} ; \mathbb{C}\right) \rightarrow H^{q}(M ; \mathbb{C}) \rightarrow H^{q-1}\left(M^{\prime \prime} ; \mathbb{C}\right) \rightarrow H^{q+1}\left(M^{\prime} ; \mathbb{C}\right) \rightarrow \cdots .
$$

Less well-known is the fact that this result extends to certain nontrivial local systems. Let $i: M \rightarrow M^{\prime}$ and $j: M^{\prime \prime} \rightarrow M^{\prime}$ be the natural inclusions and let $\mathcal{L}^{\prime}$ be a local system on $M^{\prime}$. Then there is an induced local system $\mathcal{L}=i^{*} \mathcal{L}^{\prime}$ on $M$ with trivial monodromy about the distinguished hyperplane $H$, and a local system $\mathcal{L}^{\prime \prime}=$ $j^{*} \mathcal{L}$ on $M^{\prime \prime}$ by restriction. Call $\left(\mathcal{L}, \mathcal{L}^{\prime}, \mathcal{L}^{\prime \prime}\right)$ a triple of local systems on $\left(M, M^{\prime}, M^{\prime \prime}\right)$.

Theorem 4. Let $\left(\mathcal{A}, \mathcal{A}^{\prime}, \mathcal{A}^{\prime \prime}\right)$ be a triple of arrangements, and let $\left(\mathcal{L}, \mathcal{L}^{\prime}, \mathcal{L}^{\prime \prime}\right)$ be a triple of complex rank one local systems on $\left(M, M^{\prime}, M^{\prime \prime}\right)$. Then there is a long exact sequence in local system cohomology

$$
\cdots \rightarrow H^{q}\left(M^{\prime} ; \mathcal{L}^{\prime}\right) \rightarrow H^{q}(M ; \mathcal{L}) \rightarrow H^{q-1}\left(M^{\prime \prime} ; \mathcal{L}^{\prime \prime}\right) \rightarrow H^{q+1}\left(M^{\prime} ; \mathcal{L}^{\prime}\right) \rightarrow \cdots .
$$

H. Terao informs us that this result was obtained independently by Y. Kawahara.

With the observation that $\mathcal{L}$ has trivial monodromy about the distinguished hyperplane $H$, the proof of Proposition[3]in [14] may be used to establish Theorem 4. While this result is apparently known, it does not appear that it has been recorded in the literature, and least in this form. So we provide a brief proof. More detailed arguments, in the case of trivial local coefficients, may be found in [8, 14, 19].

Proof of Theorem 4. Consider the long exact sequence of the pair $\left(M^{\prime}, M\right)$ with coefficients in $\mathcal{L}^{\prime}$. Since the local system $\mathcal{L}^{\prime}$ has trivial monodromy about the distinguished hyperplane $H$, the restriction of $\mathcal{L}^{\prime}$ to $M$ is itself a local system, $\left.\mathcal{L}^{\prime}\right|_{M}=i^{*} \mathcal{L}^{\prime}=\mathcal{L}$. So the long exact sequence of the pair is of the form

$$
\cdots \rightarrow H^{q}\left(M^{\prime}, M ; \mathcal{L}^{\prime}\right) \rightarrow H^{q}\left(M^{\prime} ; \mathcal{L}\right) \rightarrow H^{q}(M ; \mathcal{L}) \rightarrow H^{q+1}\left(M^{\prime}, M ; \mathcal{L}^{\prime}\right) \rightarrow \cdots
$$


Thus it suffices to show that $H^{q+1}\left(M^{\prime}, M ; \mathcal{L}^{\prime}\right) \cong H^{q-1}\left(M^{\prime \prime} ; \mathcal{L}^{\prime \prime}\right)$ for each $q$.

Let $E$ be a tubular neighborhood of $M^{\prime \prime}=M^{\prime} \cap H$ in $M^{\prime}$, where $H \in \mathcal{A}$ is the distinguished hyperplane. The neighborhood $E$ admits the structure of a trivial bundle over $M^{\prime \prime}$ with fiber $\mathbb{C}$. Identify the zero section of this bundle with $M^{\prime \prime}$. Then the complement of the zero section, $E_{0}$, may be identified with $E \backslash M^{\prime \prime}$.

By excision, we have $H^{*}\left(M^{\prime}, M ; \mathcal{L}^{\prime}\right) \cong H^{*}\left(E, E_{0} ; \mathcal{L}^{\prime}\right)$. Furthermore, the triviality of the bundle $E \rightarrow M^{\prime \prime}$ yields $\left(E, E_{0}\right) \cong\left(M^{\prime \prime} \times \mathbb{C}, M^{\prime \prime} \times \mathbb{C}^{*}\right) \cong M^{\prime \prime} \times\left(\mathbb{C}, \mathbb{C}^{*}\right)$. The restriction, $\left.\mathcal{L}^{\prime}\right|_{\mathbb{C}}$, of the local system $\mathcal{L}^{\prime}$ to $\left(\mathbb{C}, \mathbb{C}^{*}\right)$ is necessarily trivial. Hence, we may compute the cohomology $H^{*}\left(E, E_{0} ; \mathcal{L}^{\prime}\right)$ using the Künneth formula

$$
\begin{aligned}
H^{q+1}\left(E, E_{0} ; \mathcal{L}^{\prime}\right) & \cong H^{q+1}\left(M^{\prime \prime} \times\left(\mathbb{C}, \mathbb{C}^{*}\right) ; \mathcal{L}^{\prime}\right) \\
& \cong H^{q-1}\left(M^{\prime \prime} ;\left.\mathcal{L}^{\prime}\right|_{M^{\prime \prime}}\right) \otimes H^{2}\left(\mathbb{C}, \mathbb{C}^{*} ;\left.\mathcal{L}^{\prime}\right|_{\mathbb{C}}\right)
\end{aligned}
$$

Since $H^{2}\left(\mathbb{C}, \mathbb{C}^{*} ;\left.\mathcal{L}^{\prime}\right|_{\mathbb{C}}\right) \cong \mathbb{C}$ and the restriction of $\mathcal{L}^{\prime}$ to $M^{\prime \prime}$ is $\left.\mathcal{L}^{\prime}\right|_{M^{\prime \prime}}=j^{*} \mathcal{L}^{\prime}=\mathcal{L}^{\prime \prime}$, we have $H^{q+1}\left(M^{\prime}, M ; \mathcal{L}^{\prime}\right) \cong H^{q-1}\left(M^{\prime \prime} ; \mathcal{L}^{\prime \prime}\right)$, as was required.

Corollary 5. Let $\left(\mathcal{L}, \mathcal{L}^{\prime}, \mathcal{L}^{\prime \prime}\right)$ be a triple of complex rank one local systems on $\left(M, M^{\prime}, M^{\prime \prime}\right)$. If $H^{q-1}\left(M^{\prime \prime} ; \mathcal{L}^{\prime \prime}\right)=0$ and $H^{q}(M ; \mathcal{L}) \neq 0$, then $H^{q}\left(M^{\prime} ; \mathcal{L}^{\prime}\right) \neq 0$. If $\mathcal{L}^{\prime \prime}$ is nontrivial, then $H^{1}\left(M^{\prime} ; \mathcal{L}^{\prime}\right) \cong H^{1}(M ; \mathcal{L})$.

Proof. If $H^{q-1}\left(M^{\prime \prime} ; \mathcal{L}^{\prime \prime}\right)=0$, then the exact sequence (11) reduces to

$$
\cdots \rightarrow H^{q-2}\left(M^{\prime \prime} ; \mathcal{L}^{\prime \prime}\right) \rightarrow H^{q}\left(M^{\prime} ; \mathcal{L}^{\prime}\right) \rightarrow H^{q}(M ; \mathcal{L}) \rightarrow 0
$$

If $H^{q}(M ; \mathcal{L}) \neq 0$, then $H^{q}\left(M^{\prime} ; \mathcal{L}^{\prime}\right) \neq 0$ as well. If $q=1$ and $\mathcal{L}^{\prime \prime}$ is nontrivial, then $H^{i}\left(M^{\prime \prime} ; \mathcal{L}^{\prime \prime}\right)=0$ for $i<q$, so (2) reduces further to $H^{1}\left(M^{\prime} ; \mathcal{L}^{\prime}\right) \stackrel{\sim}{\longrightarrow} H^{1}(M ; \mathcal{L})$.

Remark 6. More general analogues of Theorem 4 include the following.

(i) Let $\mathcal{L}^{\prime}$ be a complex local system on $M^{\prime}$ of rank $m>1$, and let $\mathcal{L}=i^{*} \mathcal{L}^{\prime}$ and $\mathcal{L}^{\prime \prime}=j^{*} \mathcal{L}^{\prime}$ be the induced local systems on $M$ and $M^{\prime \prime}$. Then a straightforward modification of the argument given above yields an exact sequence,

$$
\cdots \rightarrow H^{q}\left(M^{\prime} ; \mathcal{L}^{\prime}\right) \rightarrow H^{q}(M ; \mathcal{L}) \rightarrow H^{q-1}\left(M^{\prime \prime} ; \mathcal{L}^{\prime \prime}\right) \otimes \mathbb{C}^{m} \rightarrow H^{q+1}\left(M^{\prime} ; \mathcal{L}^{\prime}\right) \rightarrow \cdots
$$

in local system cohomology generalizing (1).

(ii) [D. Massey] More generally, let $X$ be a (connected) complex analytic manifold. Let $A$ be a closed analytic subspace of $X$, and $H$ a (connected) closed submanifold of $X$ of (complex) codimension $c$ in $X$. Denote by $i: X \backslash(A \cup H) \rightarrow X \backslash A$ and $j:(X \backslash A) \cap H \rightarrow X \backslash A$ the natural inclusions.

Let $F^{\bullet}$ be a complex of sheaves on $X \backslash A$ such that the cohomology sheaves are locally constant. Thus, the sheaves $H^{k}\left(F^{\bullet}\right)$ may be nonzero in any (finite) number of degrees and may have arbitrary ranks, but, for a fixed $k, H^{k}\left(F^{\bullet}\right)$ is a local system on $X \backslash A$.

There is a fundamental distinguished triangle:

$$
j_{!} j^{!} F^{\bullet} \rightarrow F^{\bullet} \rightarrow R i_{*} i^{*} F^{\bullet} \rightarrow j_{!} j^{!} F^{\bullet}[1] .
$$

Since $j$ is the inclusion of one submanifold into another, and $F^{\bullet}$ has locally constant cohomology, there is an isomorphism $j^{!} F^{\bullet} \cong j^{*} F^{\bullet}[-2 c]$. Furthermore, for a closed inclusion, $j_{!} \cong R j_{*}$. Thus, we obtain

$$
R j_{*} j^{*} F^{\bullet}[-2 c] \rightarrow F^{\bullet} \rightarrow R i_{*} i^{*} F^{\bullet} \rightarrow\left(R j_{*} j^{*} F^{\bullet}[-2 c]\right)[1] .
$$


The associated long exact sequence in hypercohomology,

$$
\rightarrow H^{k-2 c}\left(M^{\prime \prime} ; F_{\left.\right|_{M^{\prime \prime}}}^{\bullet}\right) \rightarrow H^{k}\left(M^{\prime} ; F^{\bullet}\right) \rightarrow H^{k}\left(M ; F_{\left.\right|_{M}}^{\bullet}\right) \rightarrow H^{k-2 c+1}\left(M^{\prime \prime} ; F_{\left.\right|_{M^{\prime \prime}}}^{\bullet}\right) \rightarrow,
$$

where $M=X \backslash(A \cup H), M^{\prime}=X \backslash A$, and $M^{\prime \prime}=(X \backslash A) \cap H$, generalizes (1).

\section{TRANSLATED TORI}

We now use the results of the previous section to prove Theorem 2. For each positive integer $r \geq 2$, we exhibit an arrangement whose first characteristic variety contains one or more essential components translated by characters of order $r$.

Let $\mathcal{A}_{r}$ be the monomial arrangement of $n=3 r+3$ hyperplanes in $\mathbb{C}^{3}$ defined by $Q\left(\mathcal{A}_{r}\right)=x_{1} x_{2} x_{3}\left(x_{1}^{r}-x_{2}^{r}\right)\left(x_{1}^{r}-x_{3}^{r}\right)\left(x_{2}^{r}-x_{3}^{r}\right)$. These are the reflection arrangements associated to the full monomial groups $G(r, 1,3)$; see [14]. The characteristic varieties of these arrangements were studied in [4, §6]. There, it is shown that $\Sigma_{1}\left(\mathcal{A}_{r}\right)$ has an essential two-dimensional component $C$ which contains the identity 1. Let $\zeta=\exp (2 \pi \sqrt{-1} / r)$. Denote the coordinates of the complex torus $\left(\mathbb{C}^{*}\right)^{n}$ by $\left(z_{1}, z_{2}, z_{3}, z_{12: 1}, \ldots, z_{12: r}, z_{13: 1}, \ldots, z_{13: r}, z_{23: 1}, \ldots, z_{23: r}\right)$, where $z_{i}$ corresponds to the hyperplane $H_{i}=\operatorname{ker}\left(x_{i}\right)$ and $z_{i j: k}$ to $H_{i j: k}=\operatorname{ker}\left(x_{i}-\zeta^{k} x_{j}\right)$. In these coordinates, the component $C \subset \Sigma_{1}\left(\mathcal{A}_{r}\right)$ is given by

$$
C=\left\{\left(u^{r}, v^{r}, w^{r}, w, \ldots, w, v, \ldots, v, u, \ldots, u\right) \in\left(\mathbb{C}^{*}\right)^{n} \mid u v w=1\right\} .
$$

Notice that setting, say, $w$ equal to $\zeta^{q}$ defines a one-dimensional subvariety of $C$ which does not contain the identity, and that $z_{3}=1$ at every point in this subvariety.

In light of this observation, let $\mathcal{D}_{r}$ be the "monomial deletion" $\mathcal{A}_{r} \backslash\left\{H_{3}\right\}$, with defining polynomial $Q\left(\mathcal{D}_{r}\right)=x_{1} x_{2}\left(x_{1}^{r}-x_{2}^{r}\right)\left(x_{1}^{r}-x_{3}^{r}\right)\left(x_{2}^{r}-x_{3}^{r}\right)$. Note that $\left|\mathcal{D}_{r}\right|=$ $n-1=3 r+2$, and denote the coordinates of $\left(\mathbb{C}^{*}\right)^{n-1}$ by $z_{1}, z_{2}$, and $z_{i j: k}$, ordered as above. We assert that the subvariety $C_{q}$ of $\left(\mathbb{C}^{*}\right)^{n-1}$ defined by

$$
C_{q}=\left\{\left(u^{r}, v^{r}, \zeta^{q}, \ldots, \zeta^{q}, v, \ldots, v, u, \ldots, u\right) \in\left(\mathbb{C}^{*}\right)^{n-1} \mid u v \zeta^{q}=1\right\}
$$

is a component of the first characteristic variety $\Sigma_{1}\left(\mathcal{D}_{r}\right)$ for each $q, 1 \leq q \leq r-1$, that is clearly essential. Let $T_{q}$ be the one-dimensional subtorus of $\left(\mathbb{C}^{*}\right)^{n-1}$ given by

$$
T_{q}=\left\{\left(u^{r}, u^{-r}, 1, \ldots, 1, u^{-1}, \ldots, u^{-1}, u, \ldots, u\right) \in\left(\mathbb{C}^{*}\right)^{n-1} \mid u \in \mathbb{C}^{*}\right\},
$$

and let $\tau_{q}=\left(1,1, \zeta^{q}, \ldots, \zeta^{q}, \zeta^{-q}, \ldots, \zeta^{-q}, 1, \ldots, 1\right)$. Checking that $C_{q}=\tau_{q} \cdot T_{q}$, we see that $C_{q}$ is a translated subtorus of $\left(\mathbb{C}^{*}\right)^{n-1}$, translated by a character of order $r$.

We first establish the containment $C_{q} \subset \Sigma_{1}\left(\mathcal{D}_{r}\right)$. For this, let $\mathbf{t}^{\prime}$ be a point in $C_{q}$, given by $\mathbf{t}^{\prime}=\left(t_{1}^{\prime}, t_{2}^{\prime}, t_{12: 1}^{\prime}, \ldots, t_{12: r}^{\prime}, t_{13: 1}^{\prime}, \ldots, t_{13: r}^{\prime}, t_{23: 1}^{\prime}, \ldots, t_{23: r}^{\prime}\right)$, and let $\mathcal{L}^{\prime}$ denote the corresponding rank one local system on $M\left(\mathcal{D}_{r}\right)$. The induced local system $\mathcal{L}=i^{*} \mathcal{L}^{\prime}$ on $M\left(\mathcal{A}_{r}\right)$, where $i: M\left(\mathcal{A}_{r}\right) \rightarrow M\left(\mathcal{D}_{r}\right)$ is the inclusion, corresponds to the point $\mathbf{t} \in\left(\mathbb{C}^{*}\right)^{n}$ given by $t_{1}=t_{1}^{\prime}, t_{2}=t_{2}^{\prime}, t_{3}=1$, and $t_{i j: k}=t_{i j: k}^{\prime}$ for all $i, j$, and $k$. Since $\mathbf{t}^{\prime} \in C_{q}$, we have $\mathbf{t} \in C \subset \Sigma_{1}\left(\mathcal{A}_{r}\right)$. Thus, $H^{1}\left(M\left(\mathcal{A}_{r}\right) ; \mathcal{L}\right) \neq 0$.

Let $\mathcal{A}_{r}^{\prime \prime}=\left\{H_{3} \cap H \mid H \in \mathcal{D}_{r}\right\}$ be the restriction corresponding to the deletion $\mathcal{D}_{r}=\mathcal{A}_{r} \backslash\left\{H_{3}\right\}$. By Corollary 5 to show that $H^{1}\left(M\left(\mathcal{D}_{r}\right) ; \mathcal{L}^{\prime}\right) \neq 0$, i.e., that $\mathbf{t}^{\prime} \in$ $\Sigma_{1}\left(\mathcal{D}_{r}\right)$, it suffices to show that the local system $\mathcal{L}^{\prime \prime}=j^{*} \mathcal{L}^{\prime}$ on $M\left(\mathcal{A}_{r}^{\prime \prime}\right)$ is nontrivial, where $j: M\left(\mathcal{A}_{r}^{\prime \prime}\right) \rightarrow M\left(\mathcal{D}_{r}\right)$ is the inclusion. The hyperplanes of $\mathcal{A}_{r}^{\prime \prime} \subset H_{3}$ are $H_{1}^{\prime \prime}=H_{3} \cap H_{1} \cap H_{13: 1} \cap \cdots \cap H_{13: r}, H_{2}^{\prime \prime}=H_{3} \cap H_{2} \cap H_{23: 1} \cap \cdots \cap H_{23: r}$, and $H_{12: k}^{\prime \prime}=H_{3} \cap H_{12: k}$ for $1 \leq k \leq r$. If $\mathbf{t}^{\prime \prime}=\left(t_{1}^{\prime \prime}, t_{2}^{\prime \prime}, t_{12: 1}^{\prime \prime}, \ldots, t_{12: r}^{\prime \prime}\right) \in\left(\mathbb{C}^{*}\right)^{r+2}$ records the monodromy of $\mathcal{L}^{\prime \prime}$ about these hyperplanes, then $t_{1}^{\prime \prime}=t_{3} t_{1} t_{13: 1} \cdots t_{13: r}=1$, $t_{2}^{\prime \prime}=t_{3} t_{2} t_{23: 1} \cdots t_{23: r}=1$, and $t_{12: k}^{\prime \prime}=t_{3} t_{12: k}=\zeta^{q}$ for $1 \leq k \leq r$. Thus, $\mathcal{L}^{\prime \prime}$ is nontrivial, and $H^{1}\left(M\left(\mathcal{D}_{r}\right) ; \mathcal{L}^{\prime}\right) \neq 0$. Hence, $\mathbf{t}^{\prime} \in \Sigma_{1}\left(\mathcal{D}_{r}\right)$, and $C_{q} \subset \Sigma_{1}\left(\mathcal{D}_{r}\right)$. 
It remains to show that $C_{q}$ is a translated component of $\Sigma_{1}\left(\mathcal{D}_{r}\right)$, i.e., that $C_{q}$ is not contained in a component which passes through the identity $\mathbf{1} \in\left(\mathbb{C}^{*}\right)^{n-1}$. Suppose otherwise. Then $C_{q} \subset S \subset \Sigma_{1}\left(\mathcal{D}_{r}\right)$, and $\mathbf{1} \in S$. Since $C_{q}$ is essential, so is $S$. There is then an essential component $L \subset \mathcal{R}_{1}\left(\mathcal{D}_{r}\right)$ corresponding to $S$ by Theorem 1. However, the characterizations of the resonance varieties in [5, 12] rule out the existence of such a component $L$. For instance, it is readily checked that (the matroid of) the arrangement $\mathcal{D}_{r}$ admits no nontrivial neighborly partition. It follows that all components of $\mathcal{R}_{1}\left(\mathcal{D}_{r}\right)$ are nonessential; cf. [5, §3]. Thus, $C_{q}$ is indeed a translated component of $\Sigma_{1}\left(\mathcal{D}_{r}\right)$, which completes the proof of Theorem 2

We conclude by noting a consequence of the above discussion.

Corollary 7. For any positive integer $n$, there is a hyperplane arrangement $\mathcal{A}$ for which $\Sigma_{1}(\mathcal{A})$ contains $n$ essential positive-dimensional translated tori.

\section{ACKNOWLEDGMENTS}

We thank D. Massey for communicating to us the generalization of Theorem 4 found in Remark 6 (ii). We also thank M. Falk, P. Orlik, A. Suciu, and H. Terao for useful conversations.

\section{REFERENCES}

1. K. Aomoto, M. Kita, Hypergeometric Functions (in Japanese), Springer-Verlag, 1994.

2. D. Arapura, Geometry of cohomology support loci for local systems I, J. Alg. Geom. 6 (1997), 563-597. MR 98m:32043

3. D. Cohen, P. Orlik, Arrangements and local systems, Math. Res. Lett. 7 (2000), 299-316. MR 2001i:57040

4. D. Cohen, A. Suciu, Characteristic varieties of arrangements, Math. Proc. Cambridge Phil. Soc. 127 (1999), 33-53. MR 2000m:32036]

5. M. Falk, Arrangements and cohomology, Ann. Combin. 1 (1997), 135-157. MR 99g:52017

6. M. Falk, Combinatorial and algebraic structures in Orlik-Solomon algebras, European J. Combin. 22 (2001), 687-698. CMP 2001:16

7. I. M. Gelfand, General theory of hypergeometric functions, Soviet Math. Dokl. 33 (1986), 573-577. MR 87h:22012

8. R. Jozsa, J. Rice On the cohomology ring of hyperplane complements, Trans. Amer. Math. Soc. 113 (1991), 973-981. MR 92c:32044

9. A. Libgober, On the homology of finite abelian coverings, Topology Appl. 43 (1992), 157-166. MR 93e:57003

10. A. Libgober, Characteristic varieties of algebraic curves, in: Applications of algebraic geometry to coding theory, physics and computation (Eilat, 2001), NATO Sci. Ser. II Math. Phys. Chem., 36, Kluwer Acad. Publ., Dordrecht, 2001, pp. 215-254. CMP 2002:05

11. A. Libgober, First order deformations for rank one local systems with a non-vanishing cohomology, Topology Appl. 118 (2002), 159-168.

12. A. Libgober, S. Yuzvinsky, Cohomology of the Orlik-Solomon algebras and local systems, Compositio Math. 21 (2000), 337-361. MR 2001j:52032

13. D. Matei, A. Suciu, Cohomology rings and nilpotent quotients of real and complex arrangements, in: Arrangements - Tokyo 1998, Adv. Stud. Pure Math., vol. 27, Math. Soc. Japan, 2000, pp. 185-215. MR 2002b:32045

14. P. Orlik, H. Terao, Arrangements of hyperplanes, Grundlehren Math. Wiss., vol. 300, SpringerVerlag, 1992. MR 94e:52014

15. P. Orlik, H. Terao, Arrangements and Hypergeometric Integrals, MSJ Mem., vol. 9, Math. Soc. Japan, 2001. CMP 2001:08

16. M. Sakuma, Homology of abelian coverings of links and spatial graphs, Canad. J. Math. 47 (1995), 201-224. MR 96d:57008 
17. A. Suciu, Translated tori in the characteristic varieties of complex hyperplane arrangements, Topology Appl. 118 (2002), 209-223.

18. A. Varchenko, Multidimensional Hypergeometric Functions and Representation Theory of Lie Algebras and Quantum Groups, Adv. Ser. Math. Phys., vol. 21, World Scientific, 1995. MR 99i:32029

19. S. Yuzvinsky, Orlik-Solomon algebras in algebra and topology, Russian Math. Surveys 56 (2001), 87-166.

Department of Mathematics, Louisiana State University, Baton Rouge, Louisiana 70803

E-mail address: cohen@math.lsu.edu

URL: http://www.math.1su.edu/ cohen/ 